\title{
Development of Local Gas Temperature Measurement Technique inside a Combustion Chamber Using Two-Wire Thermocouple*
}

\author{
Shigemi KOBAYASHI ${ }^{* *}$,Yasuo MORIYOSHI ${ }^{* * *}$ and Yoshiteru ENOMOTO ${ }^{* * * *}$ \\ ${ }^{* *}$ RRD CO.,LTD. Design Department (NEDO-fellow), \\ 1-1, Ageo-shi, Saitama 362-8523, Japan \\ E-mail:shigemi_kobayashi@nissandiesel.co.jp \\ ${ }^{* * *}$ Department of Electronics and Mechanical Engineering, Chiba University, \\ 1-33, Yayoi-cho, Inage-ku, Chiba, 263-8522, Japan \\ E-mail: ymoriyos@faculty.chiba-u.jp \\ ****Department of Mechanical Systems Engineering, Musashi Institute of Technology, \\ 1-28-1, Tamazutsumi, Setagaya-ku, Tokyo, 158-8557, Japan \\ E-mail: yenomoto@sc.musashi-tech.ac.jp
}

\begin{abstract}
Thermocouples are widely used to measure the local gas temperature due to its accuracy and convenience. However, it is difficult to use thermocouples in a transient phenomenon such as reacting fields. In this study, the unsteady gas temperature inside a combustion chamber was measured by using an improved two-wire thermocouple technique. Based on previous two-wire methods, some modifications were examined. Firstly, numerical analysis of heat transfer between transient flow and thermocouple was performed to see what kind of modification was required. Secondly, a correction term was added to the basic equation, which was validated by experiments using a Rapid Compression and Expansion Machine. Finally, an improved two-wire thermocouple technique was evaluated by measuring the transient gas temperature inside a combustion chamber and compared to the estimated temperature using measured pressure data and assumptions of chemical equilibrium.
\end{abstract}

Key words: Combustion, Internal Combustion Engine, Heat Transfer, Measurement, Local Temperature, Thermocouple

\section{Introduction}

In order to investigate the details of a transient combustion phenomenon, the temporal characteristics before and after reactions are required. In particular, local information is useful to analyze the phenomenon, but the transient measurement is very difficult. Among many physical characteristics, temperature is very useful to know. Many methods for temperature measurement are available: the contacting method ${ }^{(1)}$, the method of particle number density ${ }^{(2)}$, radiation / absorption thermometry ${ }^{(3)}$, the particle excitation method ${ }^{(4)}$ and the velocity-of-sound method ${ }^{(5)}$ are available ${ }^{(6)}$. Thermocouples ${ }^{(7)}$ belong to the contacting method. Although they have good features such as a direct and continuous measurement of the local temperature change ${ }^{(1)(8)}$, simple structure and ease of handling, they have the problem of having a temporally low response. Various methods of improving the response have been examined, and thermocouples are now used for measurement and control of turbulent flow combustion fields, such as combustion and rocket research. In order to measure the gas temperature before ignition in a combustion chamber, the authors have employed a two-wire thermocouple method. In order to obtain the author's targeted objectives, the following are required i) a closed system, ii) a single-shot measurement 
starting up from static state and iii) the maximum temperature over $2000 \mathrm{~K}$ are required. Under such conditions, measurement is difficult as it is hard to estimate the temporal change of the time constant. Therefore in this work, a trial and evaluation of the new temperature measuring method has been performed. The time constant computing methods were examined using numerical analysis together. As a result, a wide range temperature change of the fuel-air mixture could be measured before and after ignition.

\section{Measurement principle}

\subsection{Basic formula}

When a hot junction is regarded sufficiently small such that the internal temperature is almost uniform, the energy balance of a hot junction will give the following formula, assuming the influence by radiation or conduction is negligible since the heat transfer on the surface is dominant.

$$
\rho c v \cdot(d T / d t)=h A \cdot\left(T_{g}-T\right)
$$

Where, $T_{g}$ is the gas temperature, $T$ is the hot junction temperature, and $h$ is the heat transfer coefficient. $\rho, c, v$, and $A$ are density of hot junction, specific heat, volume, and surface area respectively.

A coefficient $\tau$ may be defined by the following formula, which has the unit of time.

$\tau=\rho c v / h A$

If $\tau$ is substituted into Eq.(1), the basic Eq.(3) of the thermocouple method can be obtained.

$$
T_{g}=T+\tau(d T / d t)
$$

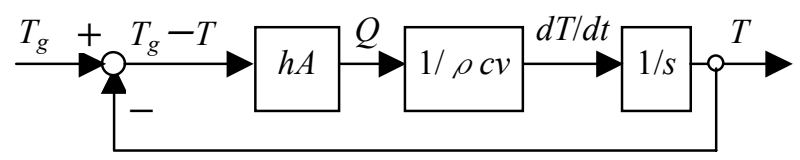

Fig.1 Block diagram of thermocouple measurement system

The temperature conversion process in a hot junction can be represented by a block-diagram in Fig.1, where $T_{g}$ is the input. A response of output $T$ is drawn from a simple closed loop, and it turns out that it is a typical first-order lag system. Here, $Q$ in the figure shows the heat transfer rate from the surrounding to a thermocouple. The constant $\tau$ in Eq.(2) determines the characteristics of a first-order lag system. It is the response determinant of a thermocouple, defined by the ratio of the thermal inertia to the heat conducting characteristic of the hot junction. When measuring temperature using a thermocouple, how to determine the time constant is quite important. Here, the heat transfer coefficient $h$ in Eq.(2) may vary, so may the time constant. Moreover, Eq.(4) equivalent to Eq.(3) can be deduced by rewriting Fig. 1 in one transfer function by equivalent conversion. (Equation (4) is deduced by making $T_{g}$ in Eq.(3) as the input.)

$$
T=1 /(1+(\rho c v / h A) s) T_{g}=1 /(1+\tau s) T_{g}
$$

Here, $s$ is the differentiation operation and $1 / s$ performs integration in the Laplace domain.

\subsection{Time constant estimation using a conventional thermocouple}

Using a conventional thermocouple, the time constant can be estimated by the following two methods.

\section{[Using an empirical formula]}

This method determines Nusselt number $N u\left(=h d_{s} / \lambda_{g}\right)$ by an empirical formula using 
material and physical properties. The averaged time constant $\tau$ is computed by $h=N u \lambda_{g} / d_{s}$ and Eq.(2). Here, $d_{s}$ represents the diameter of the hot junction and $\lambda_{g}$ the thermal conductivity of gas. For example, when the hot junction is a cylinder, $\mathrm{Nu}$ number is calculated by the following empirical formula.

$$
N u=1.11 C \cdot \operatorname{Pr}^{0.31} \cdot \operatorname{Re}^{n}
$$

where, $\operatorname{Pr}$ is Prandtl number, $R e$ Reynolds number, and $C$ and $n$ are constants obtained experimentally as a function of Reynolds number. In case of $0.4<R e<4$, for example, $C=$ 0.891 and $n=0.33$. Therefore, $h$ can be calculated by the following Eq.(6) ${ }^{(1)(9) \text {. }}$

$$
h=1.11 C u_{g}{ }^{n} d_{s}^{(n-1)} \lambda_{g}^{0.69} \mu_{g}{ }^{(0.31-n)} \rho_{g}{ }^{n} C_{p g}{ }^{0.31}
$$

Here, $u_{g}, \mu_{g}, \rho_{g}, C_{p g}$ are the flow velocity, viscosity, density and specific heat of the gas respectively.

\section{[Measuring representative $\tau$ ]}

In this method, the time constant is estimated by a step response method. The time constant estimated by this method is, however, only an averaged representative value.

Under some situations, these two methods can be adopted in practice. However, in this research, they were impossible to use because the heat transfer coefficient and hence the time constant varied due to fluctuating velocity. Meanwhile, time constants can be calculated using a couple of independent thermocouple outputs measured at the same time, even though the surrounding gas temperature and flow velocity change quickly. This is the reason why the two-wire thermocouple method is adopted in this research.

\subsection{Calculation of time constant using two-wire thermocouple}

The two-wire thermocouple method uses thermocouples with different diameters. Consider two measurement systems denoted by subscripts 1 and 2, Eq.(7) stands as a basic formula.

$$
\left.\begin{array}{l}
T_{g 1}=T_{1}+\tau_{1} \frac{d T_{1}}{d t} \\
T_{g 2}=T_{2}+\tau_{2} \frac{d T_{2}}{d t}
\end{array}\right\}
$$

The principle of the measuring method using a two-wire thermocouple is to determine each time constant so that the instantaneous temperature can be deduced. For the two hot junctions, the surrounding gas has the same physical properties, so that conditions such as temperature and flow velocity can be regarded to be the same, $\tau_{1}$ and $\tau_{2}$ in Eq. (7) will be calculated by the principle of a two-wire thermocouple. The following paragraph examines the validity of this assumption.

Methods to obtain the time constants by two-wire thermocouple have been proposed by Strahle\&Muthkrisnan ${ }^{(10)}$, Cambray ${ }^{(11)}$, and Tagawa ${ }^{(12)(13)}$ et al. The application in the turbulent flow combustion field, where temperature varies continuously, has seen much improved precision compared to a single thermocouple. The temperature computing methods of Cambray and Tagawa use transient time constants, so they are suitable for the measurements of temperature, where the time constant quickly changes corresponding to the condition in this study. As there are two methods proposed by Tagawa et al., a total of three methods are examined on the prediction of time constants below.

\section{(A)[Cambray's method $]^{(11)(14)}$}

When the basic principle of two-wire thermocouple stands, physical properties of the surrounding gas such as the flow velocity and temperature are equal around the two hot junctions. Therefore, it is assumed that the ratio of two time constants $\alpha\left(\tau_{2} / \tau_{1}\right)$ is constant. By replacing $\tau_{2}$ in Eq.(7) by $\alpha$ and $\tau_{1}$, Eq.(8) can be deduced since $T_{g l}=T_{g 2}$. 


$$
\tau_{1}=-\frac{T_{1}-T_{2}}{\left(d T_{1} / d t\right)-\alpha\left(d T_{2} / d t\right)}
$$

Once $\tau_{1}$ is solved, gas temperature can be solved by Eq.(7). It is henceforth called $\mathbf{C}_{\alpha}$ method. In this method, as the ratio of time constants are calculated directly from the diameter ratio of the thermocouple, the correct ratio of the hot junction size must be known.

Here, the ratio of time constants is computed assuming that the hot junction part is cylindrical in shape and the same diameter as the thermocouple. As $v / A$ in Eq.(2) can be simplified to $d / 4$, the ratio of time constants $\left(\tau_{2} / \tau_{1}\right)$ becomes the ratio of wire diameter multiplied by the contrary ratio of the heat transfer coefficient as in Eq.(9).

$$
\tau_{2} / \tau_{1}=\left(\rho c d_{2} /\left(4 h_{2}\right)\right) /\left(\rho c d_{1} /\left(4 h_{1}\right)\right)=\left(d_{2} / d_{1}\right) \cdot\left(h_{1} / h_{2}\right)
$$

Here, subscripts 1 and 2 show peculiar values of the $T_{1}$ and $T_{2}$ measurement systems respectively. Moreover, given that the common physical properties can be applied in Eq.(6), $h_{1} / h_{2}$ can be replaced by the wire diameter ratio in Eq.(10)

$$
\left(h_{1} / h_{2}\right)=\left(d_{1} / d_{2}\right)^{(n-1)}
$$

Therefore, using Eqs.(9) and (10), the ratio of time constants can be deduced by the ratio of thermocouple's wire diameters in Eq.(11).

$$
\tau_{2} / \tau_{1}=\left(d_{2} / d_{1}\right)^{(2-n)}
$$

\section{(B) [Tagawa's Emin method.] ${ }^{(12)}$}

Based on the assumption that $T_{g 1}$ and $T_{g 2}$ should be equal, the time constants are fixed during the time constant calculation duration. $\tau_{1}, \tau_{2}$ are determined by the condition that the value $\mathrm{E}$, which is the average of the squares of these temperature differences, is minimized in each time constant calculation duration. This method is henceforth called $\mathbf{E}_{\mathbf{m i n}}$ method.

\section{(C) [Tagawa's Rmax method.] ${ }^{(13)}$}

Based on the assumption that the fluctuating waveform of the $T_{g 1}$ and $T_{g 2}$ after compensation obtained from each thermocouple should be similar to each other, $\tau_{1}, \tau_{2}$ are determined by maximizing the correlation coefficient $\boldsymbol{R}$ of these temperature waveforms. This method assumes that the time constant is fixed in the time constant calculation duration. This method will be henceforth called $\mathbf{R}_{\max }$ method.

Although the time constants can be obtained without any geometric information on the thermocouple using the $\mathbf{E}_{\min }$ and $\mathbf{R}_{\max }$ methods, the time constants are required to be fixed for a certain amount of time duration. As the time constant can be estimated temporally by one of the methods (A) to $(\mathbf{C})$, the instantaneous gas temperature can be calculated from the hot junction temperature using Eq.(3).

Equation (5) stands that the Cambray's method assumes that the ratio of heat transfer coefficient between a thicker thermocouple and a thinner thermocouple is expressed by the ratio of wire diameters. When applying the Cambray's method in this study, Eq.(5) must be realized or $\mathrm{Nu}$ numbers of thicker thermocouple and thinner one correspond with the same power law about Re number. The effective ranges of Eq.(5) about Pr number and $\operatorname{Re}$ number are $0.6<\operatorname{Pr}<380$ and $0.4<\operatorname{Re}<4000^{(1)}$, while the experimental conditions in this study are $0.7<\operatorname{Pr}<0.8$ and $0<\operatorname{Re}<3$. Thereby, the applicability of this method was verified experimentally.

\section{2-4 Heat transfer simulation in the hot junction space}

Equation(3) is valid when the hot junction is sufficiently small, because the temperature difference between the surface layer and the core may be large in a large hot junction. As it is necessary to use the hot junction temperature as the surface temperature in Eq.(3), the average temperature measured by the hot junction is not appropriate. However, checking the 


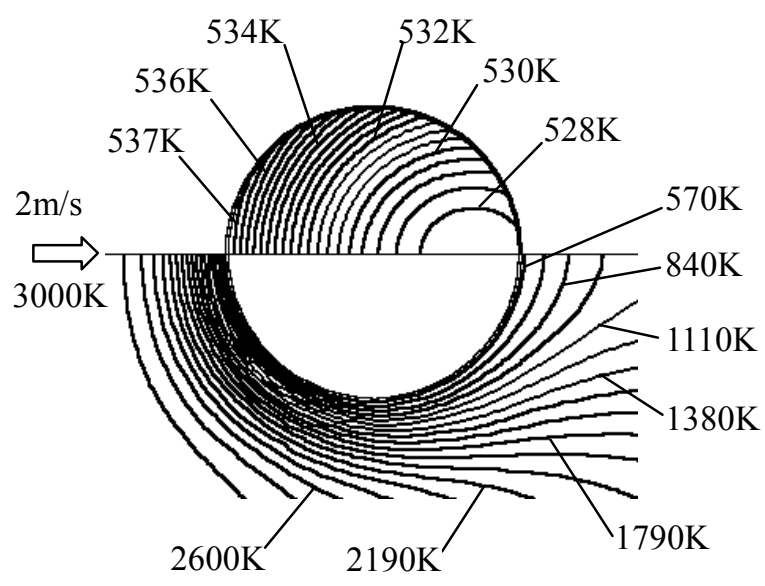

Fig.2 Calculated temperature range inside and outside thermocouple $\phi 100 \mu \mathrm{m}$

temperature distribution inside the hot junction is difficult, so a thermocouple that receives a high temperature gas flow was numerically simulated.

An air flow of $3000 \mathrm{~K}$ was made to blow at $2 \mathrm{~m} / \mathrm{s}$ to the $100 \mu \mathrm{m}$ wire at room temperature. The temperature distribution of the wire and flow field were calculated in 2D using a commercial code FLUENT6.1. The physical-properties of Chromel were used for that of the solid cylinder, e.g. thermal conductivity. The calculation was performed using fine calculation grids (the size of the calculation grid is about $2.5 \mu \mathrm{m}$ ). The boundary conditions of the simulation were set to allow for potentially large temperature variation inside the hot junction in the combustion vessel. The temperature distributions of both the interior of the wire and the surrounding gas after $10 \mathrm{~ms}$ of gas was blown is shown in the upper and lower frames of Fig. 2 respectively. Although the temperature range inside the wire is no more than $10 \mathrm{~K}$, the temperature range of the surrounding gas is as large as about $2400 \mathrm{~K}$. The center of the contours inside the wire shifts towards downstream evidently, and the minimum temperature range appears on the surface of the hot junction. In other words, a high temperature part wraps a low temperature part in it. It is confirmed that the influence of a temperature distribution of surrounding gas is stronger than the influence of the size of a hot junction. Therefore, when the gas passes by the hot junction, its temperature falls. When the gas flows reversely towards the hot junction again, the accuracy of the estimated gas temperature will be worse.

When considering a step temperature input from $300 \mathrm{~K}$ to $3000 \mathrm{~K}$ in Fig.2, the time-constant $\tau$ which can be derived from Eq.(2) corresponds to the time to reach $63.2 \%$ of the final value. In this condition, the time constant is estimated at $110 \mathrm{~ms}$ using the physical properties of air and thermocouples. Using Eq.(14) that appears later, the temperature after $10 \mathrm{~ms}$ takes $534.6 \mathrm{~K}$ using $T_{g(i)}=3000 \mathrm{~K}, T_{(i-1)}=300 \mathrm{~K}$, and $\tau_{(i)}=0.110$ s. This corresponds to the temperature range of $528 \mathrm{~K}-537 \mathrm{~K}$ indicated in Fig.2. Thereby, the predicted wire temperature is proved appropriate.

In this simulation shown in Fig.2, the temperature variation is less than $\pm 5 \mathrm{~K}$ to the mean temperature. Furthermore, either when more time elapses, the hot junction is smaller, or the gas flow velocity is smaller, the temperature variation become smaller than this condition. Therefore, it can be concluded that the size of the hot junction examined in this simulation was sufficiently small for applying Eq.(3).

On the other hand, the sensitivity of the distance between the two hot junctions of two wires should be investigated for this two-wire system. The distance between the hot junctions has a minimum limit, which is determined by the interference of each thermal boundary layer, while the maximum limit should be determined by the condition that the 

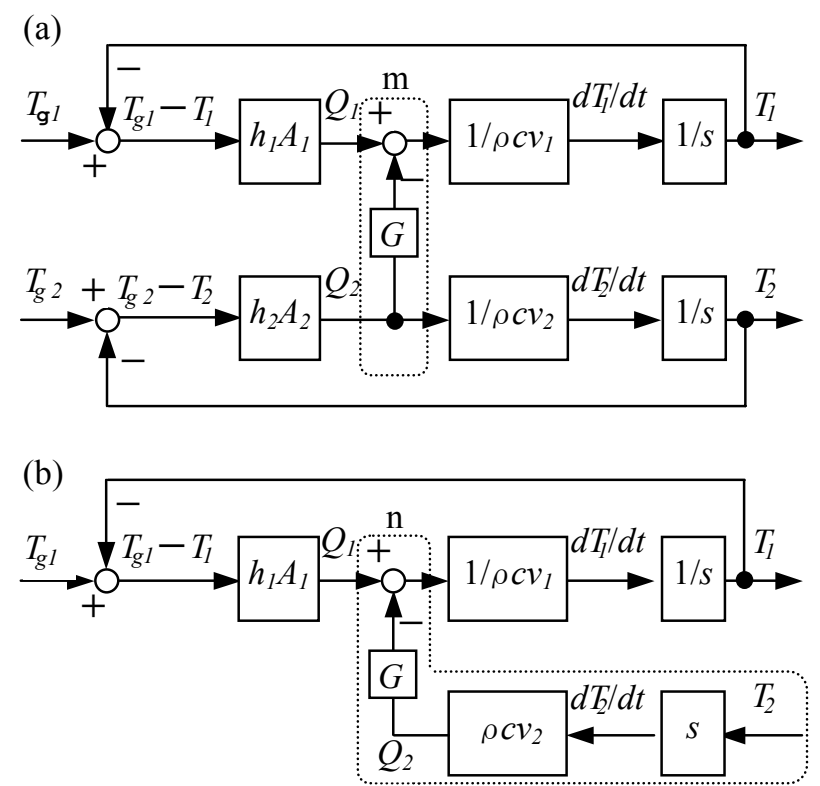

Fig.3 Block diagram of two-wire thermocouple measurement system

two hot junctions have the same gas temperature. Although the prediction of the maximum limit is difficult, that of the minimum is possible by simulation. Therefore, when designing and making a two-wire thermocouple, the gap between hot junctions was set to the size of the larger wire diameter which was regarded as the minimum limit.

\section{2-5 The compensation method of $T_{I}$ measurement system with a smaller diameter}

As mentioned, the thermocouple used in this research was made so that the gap between the hot junctions was equal to the size of the larger wire diameter. However, since the flow condition of the gas changes broadly, a compensation method was examined that assumed that the thinner thermocouple was indeed affected by the thermal boundary layer of the thicker thermocouple.

Figure3(a) illustrates the measurement system of a two-wire thermocouple in a block diagram like Fig.1. Assuming that the quantity of heat transfer by the thicker wire influences that by the thinner wire, $T_{1}$ and $T_{2}$ measurement systems are combined in this diagram. The dashed line part $[\mathrm{m}]$ in the figure shows a joint portion, and the transfer course in which $T_{2}$ measurement system influences $T_{1}$ measurement system via transfer function $G$ through quantity of heat transfer $Q$ is added. Since there is no influence by the addition of joint portion in $T_{2}$ measurement system (the wire with the thicker diameter), the block diagram is the same as of Fig.1, and the relation of input and output can be expressed by Eq.(3).

Based on Fig.3(a), $T_{T}$ measurement system can be represented in Fig.3(b). It is a two-input system in which the dashed line part $[\mathrm{n}]$ is added to a first-order lag system. Equivalent conversion of a block diagram has been performed using the principle of superposition after drawing the input and output relation from the two inputs ( $T_{g 1}$ and $\left.T_{2}\right)$ to a single output $T_{1}$. Eq.(12) can be obtained by solving for $T_{g l}$.

$$
T_{g l}=\left(1+\tau_{l} \cdot s\right) \cdot T_{l}+G\left(\rho c v_{2} / h_{l} A_{l}\right) \cdot s T_{2}
$$

Therefore, when the heat exchange of the thinner wire is influenced by the heat transfer state of the thicker wire, it is necessary to use Eq.(13) instead of Eq.(7).

$$
\left.\begin{array}{l}
T_{g 1}=T_{1}+\tau_{1} \frac{d T_{1}}{d t}+G \frac{\rho c v_{2}}{h_{1} A_{1}} \times \frac{d T_{2}}{d t} \\
T_{g 2}=T_{2}+\tau_{2} \frac{d T_{2}}{d t}
\end{array}\right\}
$$


The difference from Eq.(7) is the third term on the right side added to $T_{l}$ measurement system, and will have rectified the mismatching of $T_{l}$ measurement system using $T_{2}$ measured value. Here, the coefficient of $G\left(=\rho c v_{2} / h_{1} A_{1}\right)$ is called a compensation coefficient $G$, since Eq.(7) is equal to Eq.(13) when $C_{f}=0$. As the compensation coefficient $C$ value is given according to the heat transfer situation between the two wires, Eq.(13) is valid as the basic formula of temperature calculation.

3. Examination of the temperature calculation method using virtual hot junction's temperature

The purpose of this section is to investigate the temperatures obtained by different computing methods using the hot junction's temperature, which can be theoretically found using an idealized standard temperature pattern and a certain predefined time constant condition before applying these methods to experiment. Supposing there is a standard temperature pattern: a gas is initially held at room temperature. It shifts to a oscillating state at a certain time. An oscillatory waveform was taken as a sine wave with an amplitude of $1000 \mathrm{~K}$ and a neutral position of $1300 \mathrm{~K}$. The hot junction's temperature was calculated from a standard temperature wave pattern for every sampling cycle ( $\Delta t)$ using Eq.(14), which was rewritten from Eq.(3), into a recursive formula for step-by-step calculations.

$$
T_{(i)}=T_{g(i)}-\left(T_{g(i)}-T_{(i-1)}\right) \cdot e^{(-\Delta t / \tau(i))}
$$

Here, $T_{g(i)}$ and $T_{(i)}$ are the gas temperature and the hot junction temperature in the i-th sampling time respectively. $\tau_{(i)}$ is the time constant changing for every $\Delta t$. Considering three simplified cases in which the rate of change of $\tau$ with respect to time $d \tau / d t$ was zero, positive, and negative, and that the time constant ratio was 3.18 (equivalent to wire diameter ratio $1: 2$ ), the hot junction temperatures of $T_{1}$ and $T_{2}$ were computed numerically.

(a) $d \tau / d t=\mathbf{0}$
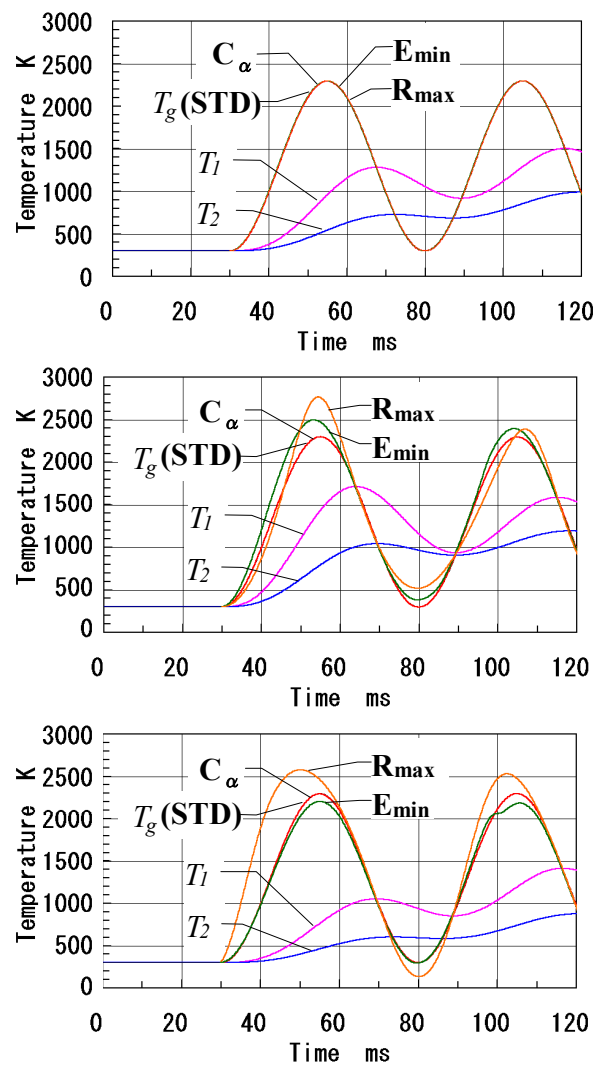

Fig.4 Compensated temperatures using various methods with virtual hot-junction temperature 
$T_{1}$ and $T_{2}$ are plotted together with the compensated temperature for each time constant condition in Fig.4 (a) - (c).

Fig.4(a) is the case when $d \tau / d t=0$, and hence the time constants are fixed ( $\tau_{1}=30 \mathrm{ms,}$ $\tau_{2}=90 \mathrm{~ms}$ always). Fig.4(b) is the case of $d \tau / d t>0$, and the time constants increase gradually $\left(\tau_{1}=10 \mathrm{~ms} \rightarrow 50 \mathrm{~ms}, \quad \tau_{2}=30 \mathrm{~ms} \rightarrow 150 \mathrm{~ms}\right.$ ). Fig.4(c) is the case of gradually decreasing time constant $d \tau / d t<0,\left(\tau_{1}=50 \mathrm{~ms} \rightarrow 10 \mathrm{~ms}, \quad \tau_{2}=150 \mathrm{~ms} \rightarrow 30 \mathrm{~ms}\right)$. Here, since the $\mathbf{E}_{\min }$ and $\mathbf{R}_{\mathbf{m a x}}$ methods require a finite duration for time constant calculation, the duration was set to $60 \mathrm{~ms}$.

Only the $\mathbf{C}_{\alpha}$ method can similarly reproduce to the standard temperature pattern at all time in all three time constant conditions. In cases of the $\mathbf{E}_{\min }$ and $\mathbf{R}_{\mathbf{m a x}}$ methods, an error appeared at the first crest of the temperature waves when the time constant was increasing or decreasing. See Fig.4(b) or 4(c).

This depended on whether the time constant calculation duration was required or not. In this research, as single-shot (transient) temperature fluctuations must be measured, the first temperature wave must be computed correctly. The $\mathbf{C}_{\alpha}$ method was employed for temperature measurement in this study because the accuracy of the first wave was higher than using other methods.

A larger noise (an artificial noise consisting of sine wave at a frequency of $1 \mathrm{kHz}$, and $10 \mathrm{~K}$ amplitude) was superimposed on the virtual temperatures of the hot junctions. Although the error tended to increase using any of the methods ( $\pm 10 \%$ of maximum), there is not much difference among the three methods. However, unexpected noise may drive the denominator of Eq.(8) near zero in the temperature calculation process causing the computed temperature to become unstable and diverge. Therefore, it is necessary to reduce noise in the measured raw temperature of the hot junction as much as possible.

\section{Measurement Devices}

\section{4-1 Structure of heat sensing part}

The tip of the two-wire thermocouple is shown in Fig.5. Two thermocouple wires were crossed-over with a certain gap using four struts.

Chromel and Alumel wires (K-type) of $50 \mu \mathrm{m}$ and $100 \mu \mathrm{m}$ diameter were used. The hot junction was made by percussion welding, so that the diameter of the junction was the same as the wire. In other words, a uniform cross section was maintained along the thermocouple wire. The thermometer wires were arranged in a cross configuration in order to keep the thermal radiation between the two wires small. Each thermocouple was welded by silver alloy to the strut (diameter of $0.6 \mathrm{~mm}$ ), which was made of the same material as the thermocouple. Based on the simulation results, the gap between the hot junctions was set to $0.1 \mathrm{~mm}$, which was the shortest distance. Moreover, in order to maintain sufficiently small influence of heat conduction in the wire axial direction, the thermocouple wire was set to $8 \mathrm{~mm}, 160$ times (for $50 \mu \mathrm{m}$ wire) or 80 times (for $100 \mu \mathrm{m}$ wire) larger than the wire diameter. The authors selected the thinnest wire diameter to be $50 \mu \mathrm{m}$ because a

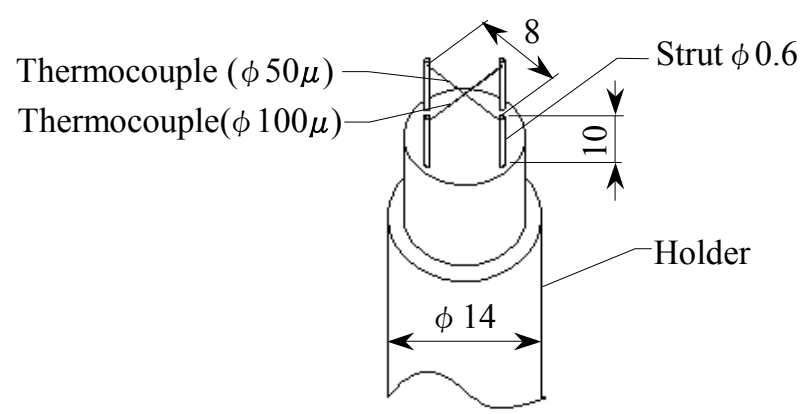

Fig.5 Structure of two-wire thermocouple 


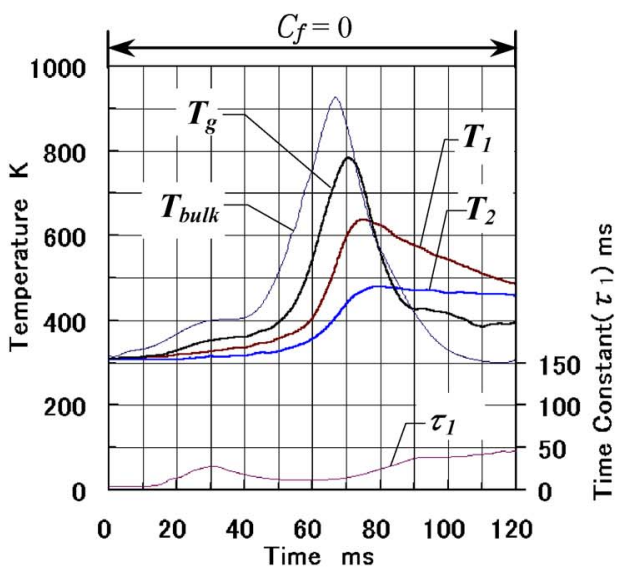

Fig.6(a) Measured Temperature in RCEM

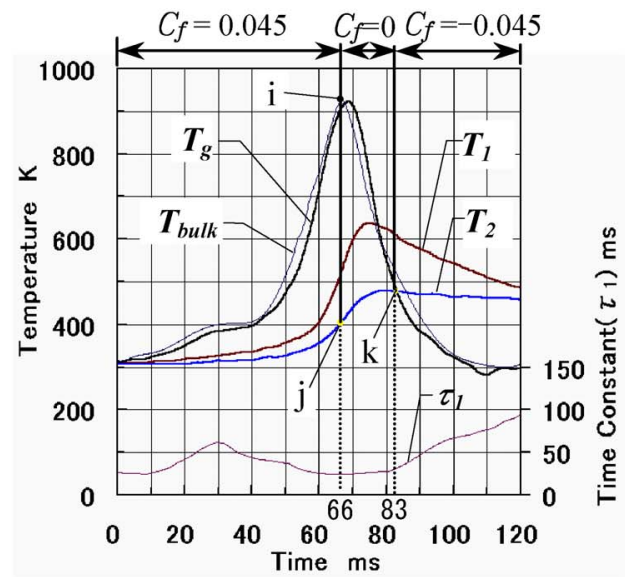

Fig.6(b) Measured Temperature in RCEM

thermocouple with a diameter of $25 \mu \mathrm{m}$ would melt easily in combustion.

\section{4-2 Temperature measurement circuit}

The electromotive forces generated by each thermocouple that has different thermal inertia were observed with a digital oscilloscope. The sampled voltages of each thermocouple output were transferred to a PC. These were then converted into a hot junction temperature by fitting it with a polynomial expression. Finally, the time constants and the gas temperature were calculated using a temperature calculation program based on the measurement principles described.

\section{Measurement in transient temperature field without combustion}

\section{5-1 Experimental set up and procedure}

The two-wire thermocouple methods can be verified using a Rapid Compression and Expansion Machine $^{(15)}$ (weight-falling-by-gravity-type). As temperature inside the cylinder may be regarded as almost uniform except near the wall, therefore a bulk temperature can be thermodynamically calculated from the measured pressure assuming adiabatic change. The computed temperatures derived from the two-wire thermocouple can be compared against the bulk temperature derived from the pressure measurements, so evaluating the accuracy of the measurement is possible.

\section{5-2 Data processing and the result}

Figures 6(a) and 6(b) are the results of the computed gas temperature using the two-wire thermocouple with and without using the compensation coefficients. In these figures, the two hot junctions' temperatures $T_{1}(\phi=50 \mu \mathrm{m})$ and $T_{2}(\phi=100 \mu \mathrm{m})$, fluctuating time constant $\tau_{1}$ obtained from $T_{1}$ and $T_{2}$, the gas temperature $T_{g}$, and the bulk temperature $T_{b u l k}$ are indicated. ( $\tau_{2}$ is not shown, since $\tau_{2}$ is just a multiple of $\tau_{1}$ scaled by the time constant ratio). For this RCEM, as the reproducibility of the piston position with time is not sufficiently high and measuring the position is not easy, ideal gas law was not used for the calculation of bulk temperature. Instead the following equation assuming adiabatic change was used:

$$
T_{\text {bulk }}=T_{0}\left(P / P_{0}\right)^{(\kappa-1) / \kappa}
$$

Here, as specific heat ratio $\kappa$ is a function of temperature, it was updated step by step using the latest temperature value computed at every time step.

Initially, when the temperature calculation was carried out with the compensation coefficient $C_{f}=0$ in Eq.(13), the gas temperature was found to be 50 to $150 \mathrm{~K}$ lower than the bulk temperature as shown in Fig.6(a). For example, the peak of the bulk temperature 
was about $940 \mathrm{~K}$ while the peak of the estimated temperature was about $780 \mathrm{~K}$.

Therefore, temperature calculation was performed using the compensation coefficient $C_{f}$ examined in Section 2.5. ( $C_{f}$ was set at 0.045$)$ so that the peak value of the calculated temperature agreed with the bulk temperature. This compensation was applied to the period from $\mathrm{t}=0$ to $66 \mathrm{~ms}$, which corresponded to the end of the compression. The calculated result indicated in Fig.6(b) showed good agreement with the bulk temperature over the entire compression period. The maximum difference between the two temperature was $77 \mathrm{~K}$ ( $8 \%$ ) at around 50 to $60 \mathrm{~ms}$ when the temporal variation of temperature was large. In contrast, the difference was less than $20 \mathrm{~K}$ ( $2 \%$ ) at the period when the temporal variation of temperature was relatively small. For setting the compensation period, either the maximum point of bulk temperature [point $\mathbf{i}$ in Fig.6(b)] or the inflection point of hot junction temperature [point $\mathbf{j}$ in Fig.6(b)] can be used. This means that the compensation period can be determined by the raw temperature.

The reason why compensation is needed in the compression process is probably because the thicker wire had a thicker boundary layer which affected the boundary layer of the thinner wire. The thickness of a thermal boundary layer is influenced by the temperature difference between the hot junction and the surrounding gas as well as the flow velocity around the junction. When the difference between the hot junction's temperature at the start time of measurement and the maximum gas temperature of the target is large, the effect of thermal inertia becomes large. This is enhanced in single-shot measurement starting up from static state, and is more severe compared to the continuous measurements of continual phenomenon.

Apart from the compression period, using a compensation coefficient is also necessary in the expansion period, when the gas temperature falls more than the hot junction temperature. The compensation coefficient $C_{f}$ was set to -0.045 (opposite-signed to the compression). As shown in Fig.6(b), it was assigned for $\mathrm{t}=83 \mathrm{~ms}$ or later period when the gas temperature is less than the hot junction temperature of the thicker wire. Consequently, the calculated temperature agreed with the bulk temperature. Here, it should be noted that the compensation period can again be judged from the hot junction data. Equation(3) indicates that the necessary and sufficient condition for $T_{g}=T$ is $d T / d t=0$. When $d T_{2} / d t=0$ occurs, the gas temperature becomes equal to the hot junction temperature of the thicker wire. Figure 6(b) shows that the compensation period can be determined by the hot junction data alone; $\mathrm{t}=83 \mathrm{~ms}$ [point $\mathbf{k}$ in Fig.6(b)] is the beginning of compensation when $d T_{2} / d t=$ 0 . 


\section{Measurement with combustion in a high temperature field}

\section{6-1 Experimental apparatus and the procedure}

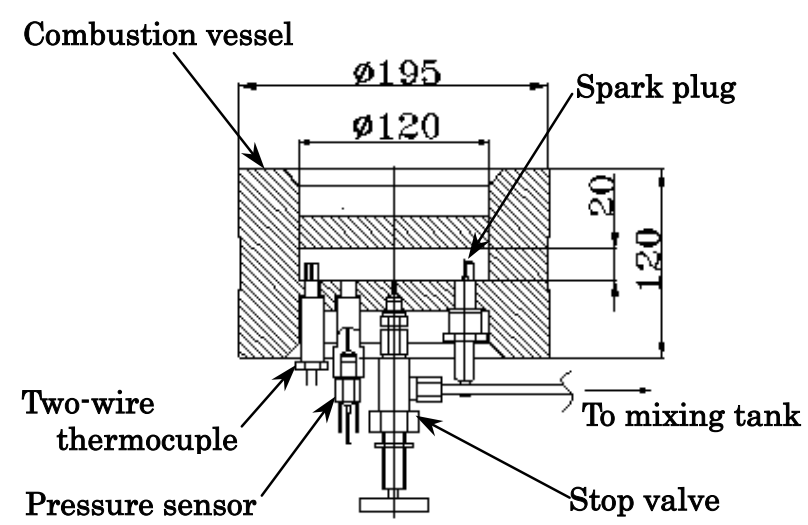

Fig.7 Combustion vessel

The outline of the combustion vessel employed in this study is shown in Fig.7. This vessel is a pancake-shaped chamber (of volume $226 \mathrm{cc}$ ) with a diameter of $120 \mathrm{~mm}$ and a height of $20 \mathrm{~mm}$. In order to preheat the fuel-air mixture to the desired temperature, electric heaters were installed in all walls enclosing the chamber, and a spark plug was located in a position $45 \mathrm{~mm}$ off center of the chamber. The two-wire thermocouple was installed on the peripheral wall, $97 \mathrm{~mm}$ left of the ignition plug, and the vertical position was set at the center of the chamber. As this measurement position was $8 \mathrm{~mm}$ away from the side wall, a measurement inside the end gas region could be made. A pressure sensor (Kistler 601B) and a valve for charging fuel-air mixture were installed on the bottom of the chamber. For these experiments, the chamber was evacuated, and then filled with a mixture of n-pentane and air with an equivalence ratio of 0.35 at a pressure of $0.3 \mathrm{MPa}$. Argon was used as a diluent to increase the burned gas temperature. The molar concentration ratio of oxygen (oxidizer) to argon was set at 0.4 to enhance autoignition.

\section{6-2 Experimental results and discussion}

Figure 8 shows the temperature history of end-gas $\left(T_{g}\right)$ from spark ignition when the wall temperature of the chamber was adjusted to $473 \mathrm{~K}$. In this figure, the bulk temperature $T_{\text {bulk }}$ calculated from pressure is also indicated. The compensation of temperature was made to the point of inflection of $T_{2}$ (point $l$ in the figure) using $C_{f}=0.045$. Moreover, because the signal to noise $(\mathrm{S} / \mathrm{N})$ ratio of the thermocouple output was low before the flame had reached the hot junction, the calculation length $(w)$ for differentiation calculation was set to $4 \mathrm{~ms}$. After that, $w$ was set to $0.4 \mathrm{~ms}$. The measured temperature was close to the bulk temperature as a result. When the calculation length was set to $4 \mathrm{~ms}$ all the time, the computed temperature was too high compared to the bulk temperature. The measured maximum temperature of $2780 \mathrm{~K}$ was close within $3 \%$ to the adiabatic equilibrium flame temperature $2710 \mathrm{~K}$ by the computing program ${ }^{(16)(17)}$. This means that the applied compensation coefficient is appropriate. The gradual temperature rise after spark ignition is considered to be due to the compression of the unburned gas by the expansion of burned gas, i.e. flame propagation. Assuming that the unburned gas was compressed uniformly and adiabatically, the burned gas temperature was estimated. When the measured temperature $T_{g}$ is compared with the calculated temperature using the averaged ratio of specific heat ${ }^{(16)}$ ( $\kappa=1.44$ ) between $473 \mathrm{~K}$ and $700 \mathrm{~K}, T_{g}$ temperature (adiabatic curve in a figure) tends to be higher than the calculated one, even though they are in agreement at the beginning of 


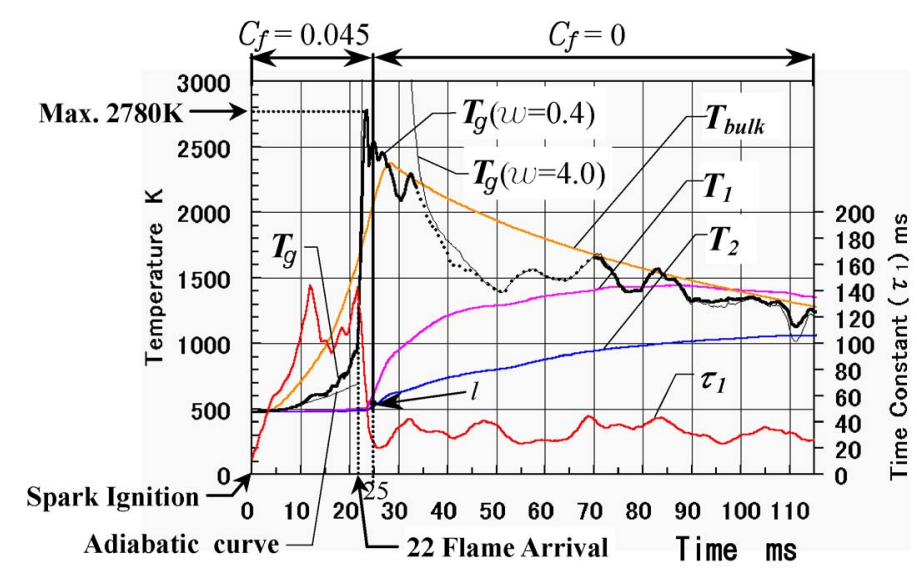

Fig.8 Measured temperature in combustion vessel

compression. As the temperature of the unburned gas is raised up to $600 \mathrm{~K}-700 \mathrm{~K}$, at which low-temperature oxidation reactions start, it releases heat locally. A rapid temperature rise was measured at around $t=22 \mathrm{~ms}$ when the flame front had arrived.

Thereafter, a temperature plateau was found at around $t=50 \mathrm{~ms}$. Regarding the flow field around the thermocouple, a flow should have come from the spark plug when the flame was propagating. However, once the flame front had passed the hot junction, the flow field could have changed completely. This affects the measurement accuracy as found in the numerical simulation.

Lastly, in order to consider the influence of thermal radiation, the radiative heat transfer rate was estimated between the chamber wall and the hot junction, the high temperature gas and the hot junction, and between the two hot junctions. The results show that the thermal radiation between chamber wall and hot junction was dominant, but the effect was at most $2 \%$ to the measured temperature. Thereby, the temperature calculation was performed without accounting for the thermal radiation.

\section{Conclusions}

In order to measure the local gas temperature inside a combustion chamber, two-wire thermocouple method was developed. The following conclusions can be deduced.

(1) An investigation on different time-constant computing methods was carried out. As a result, the method, which does not require the time-constant calculation period, is suitable for measuring such a special temperature field with sufficient accuracy where the time-constant rapidly fluctuates.

(2) The interior of the hot junction of a thermocouple and the temperature distribution of surrounding gas were numerically analysed. It was found that the internal temperature variation of the thermocouple was less than $\pm 5 \mathrm{~K}$. The conditions were: $100 \mu \mathrm{m}$ junction size, an initial gas temperature of over $300 \mathrm{~K}$, a combustion gas temperature of $3000 \mathrm{~K}$, and a gas flow velocity of $2 \mathrm{~m} / \mathrm{s}$ or less at the time of flame front approach. This means it is valid to apply the basic equation of a thermocouple Eq.(3).

(3) It is necessary to determine the distance between the two wires carefully. The distance should be small enough to achieve a point measurement, and also to satisfy the condition that the two hot junctions are subject to the same gas temperature. At the same time, the distance should be equal to or larger than the larger wire diameter.

(4) Temperature measurements were performed using a RCEM with no combustion. A large error arose during the compression stroke when there was a large temperature difference between the hot junction and the surrounding gas. This is because the heat transfer to the hot junction of the thinner wire was affected by the heat transfer to the hot junction of the 
thicker wire. This can be compensated by adding a term to the basic equation.

(5) The measurement accuracy of the gas temperature using the compensation coefficient during compression and expansion was within $8 \%$ of the bulk temperature.

(6) A local temperature measurement was performed in a combustion field inside a constant-volume vessel using the two-wire thermocouple. By applying the compensation coefficient obtained from the RCEM experiment, the gas temperature before ignition could be measured. Moreover, the temperature after ignition was also measurable except for a brief period.

The authors would like to express appreciation to the staff at Enomoto's laboratory who assisted in the manufacture of the unique thermocouple with a constant cross section along wire axis. All related companies for supplying equipment for this study are acknowledged.

\section{References}

(1) The Japan Society of Mechanical Engineers ed., JSME Standards: The Engine Temperature Measurement (in Japanese), JSME S007, (1986), The Japan Society of Mechanical Engineers.

(2) A.G.Gaydon, Flames, 4th edition, (1978), p.304, Chapman and Hall.

(3) Matsui, Kamimoto, Matsuoka and Oguri, A Study on Measurement of Flame Temperature in the Diesel Engine (in Japanese), Transactions of the Japan Society of Mechanical Engineers, Vol.44,No.377, (1978), pp.228-238.

(4) The Society of Chemical Engineers Japan ed., Combustion \&Thermal Engineering (in Japanese), (1987), p83, Maki Shoten.

(5) Mine, Combustion Study (in Japanese),No.76,(1987), Combustion Society of Japan.

(6) Mizutani,H., Combustion Engineering, (2002),pp.235-244, Morikita Publishing Co., Ltd.

(7) Japanese Industrial Standards Committee ed., JIS C1602: Thermocouples,(1995),JSA.

(8) Mizutani,H., Matsumoto,Y.,and Katsuki,M., Measurement of Flame Temperature and Fluctuation using thermocouple (in Japanese), Transactions of the Japan Society of Mechanical Engineers, Series B,Vol.51,No.468,(1985),pp.2656-2663.

(9)Uchida, Heat Transfer,(1980),p148, Shokabo Publishing Co., Ltd.

(10) Warren C.Strahle , M.Muthkrishnan, AIAA Journal,14-11,(1976),pp.1642-1644.

(11) P.Cambray, Measuring thermocouple time constants: A new method, Combustion Science and Technology, Vol.45, (1986), pp.221-224

(12) Tagawa,M., Okuda,M. and Ohta,Y., Two-wire Thermocouple for Fluctuating Temperature Measurements in Combustion, Transactions of the Japan Society of Mechanical Engineers, Series B,Vol.62, No.598, (1986), pp.2506-2512.

(13) Tagawa,M., Shimoji,T. and Ohta,Y., Improvement of a Two-Thermocouple Probe Technique for Fluctuating Temperature Measurement, Transactions of the Japan Society of Mechanical Engineers., Series B, Vol.64, No.625, (1998), pp.3077-3083

(14) M.Vachon, P.Cambray, T.Maciaszek and J.C.Bellet, Temperature and Velocity Fluctuation Measurements in a Diffusion Flame with Large Buoyancy Effects, Combustion Science and Technology, Vol.48,(1986),pp.223-240.

(15) Kobayashi,S., Moriyoshi,Y. and Enomoto,Y., Auto-ignition of Premixed Mixture Using a Constant Volume Vessel, Proceedings of 17th Internal Combustion Engine Symposium,Japan, (2002-10), pp.89-94.

(16) Bonnie J.McBride and Sanford Gordon, NASA Reference Publication 1311 Computer Program for Calculation of Complex Chemical Equilibrium Compositions and Applications, (1996), pp.1-178, NASA.

(17) Koshi,M., Low Temperature Oxidation Reaction and High Temperature Oxidation Reaction of Fuel (in Japanese),JSME Learning course Text: Basics and applications for Engine Experiment and Measurement No.6 (in Japanese),No.02-84,(2002-11), pp.27-32 